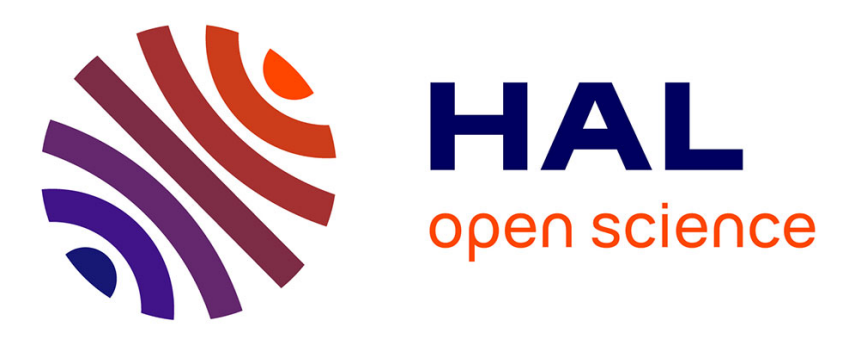

\title{
Sub-micro a-C:H patterning of silicon surfaces assisted by atmospheric-pressure plasma-enhanced chemical vapor deposition
}

Alexis Boileau, Thomas Gries, Cédric Noel, Rodrigo Perito Cardoso, Thierry Belmonte

\section{To cite this version:}

Alexis Boileau, Thomas Gries, Cédric Noel, Rodrigo Perito Cardoso, Thierry Belmonte. Sub-micro a-C:H patterning of silicon surfaces assisted by atmospheric-pressure plasma-enhanced chemical vapor deposition. Journal of Physics D: Applied Physics, 2016, 49 (44), pp.445306. 10.1088/00223727/49/44/445306 . hal-02113592

\section{HAL Id: hal-02113592 \\ https://hal.science/hal-02113592}

Submitted on 14 May 2019

HAL is a multi-disciplinary open access archive for the deposit and dissemination of scientific research documents, whether they are published or not. The documents may come from teaching and research institutions in France or abroad, or from public or private research centers.
L'archive ouverte pluridisciplinaire HAL, est destinée au dépôt et à la diffusion de documents scientifiques de niveau recherche, publiés ou non, émanant des établissements d'enseignement et de recherche français ou étrangers, des laboratoires publics ou privés. 


\title{
Sub-micro a-C:H patterning of silicon surfaces assisted by atmospheric pressure plasma-assisted chemical vapor deposition
}

Alexis Boileau ${ }^{1}$, Thomas Gries ${ }^{1}$, Cédric Noël ${ }^{1}$, Rodrigo Perito Cardoso ${ }^{2}$ and Thierry Belmonte $^{1, *}$.

${ }^{1}$ Université de Lorraine, Institut Jean Lamour, Département CP2S, UMR CNRS 7198, Parc de Saurupt, Nancy, F-54011, France.

${ }^{2}$ Universidade Federal do Paraná, Setor de Tecnologia, Departamento de Engenharia Macânica, Curitiba, Brazil

*E-mail: thierry.belmonte@univ-lorraine.fr

Keywords: a-C:H, micro and nano-patterning, dewetting, afterglow, 3D printer

\begin{abstract}
Micro and nano-patterning of surfaces is a trend challenge in the field of the miniaturization of devices assembled via top-down approaches. This study demonstrates the possibility of depositing sub-micrometric localized coatings - spots, lines or even more complex shapes - made of amorphous hydrogenated carbon $(\mathrm{a}-\mathrm{C}: \mathrm{H})$ thanks to a moving $\mathrm{XY}$ stage. Deposition was performed on silicon substrates using chemical vapor deposition assisted by an argon atmospheric pressure plasma jet (APPJ). Acetylene was injected into the post-discharge region as precursor by means of a glass capillary with a sub-micrometric diameter. A parametric study was carried out to study the influence of the geometric capillary configurations (capillary diameter and capillary-plasma distance) on the deposited coating. Thus-formed patterns were investigated by Scanning Electronic Microscopy and Atomic Force Microscopy. Furthermore, chemical composition of large coated areas was investigated by Fourier Transformed infrared spectroscopy according to the chosen atmospheric
\end{abstract}


environment. Observed chemical bonds show that reactions of the gaseous precursor in the discharge region and both chemical and morphological stability of the patterns after treatment are strongly dependent on the surrounding gas. Various sub-micrometric a-C:H shapes were successfully deposited under controlled atmospheric conditions using argon as inerting gas. Overall, this new process of additive manufacturing by atmospheric plasma offers an unusually high resolution at low cost. 


\section{Introduction}

Micro and nano-structuration of surfaces is a topical challenge in the field of ultrafine process applications. Miniaturization of devices increases the need of bottom-up or down-to approaches for their integration in lab-on-chips, Micro-Electro Mechanical Systems (MEMS) or for applications in photonic, medicine, biology, micro-fluidic or watch-making domains. The additive and subtractive approaches compete currently with each other. Even so, the additive approach seems to be the most efficient tool for extreme miniaturization, limiting by the way material losses. Among the common additive microfabrication techniques such as Physical Vapor Deposition, Plasma-Enhanced Chemical Vapor Deposition, Molecular Beam Epitaxy or 3D laser micro-lithography, the resolution is often restricted to a limited scale range and/or dedicated to some materials only. For instance, the two-photon polymerization (2PP) is a very attractive technique among the aforementioned examples [Maruo (1997), Cumpston (1999), Kawata (2001), Serbin (2003), Deubel (2004)]. Three-dimensional microstructuring of photosensitive materials by $2 \mathrm{PP}$ is effective for the fabrication of $3 \mathrm{D}$ structures having a resolution of $200 \mathrm{~nm}$ or higher $(100 \mathrm{~nm})$ [Serbin (2003)]. The 2PP approach provides much better structural resolution and quality than stereo-lithography. However, these two processes are only dedicated to the microfabrication of exclusive photosensitive materials and cannot be considered for carbon polymers and other materials as oxides or metals. Consequently, the main strategic stake consists in the development of a new 3D micro and nano-fabrication process with high flexibility of use, wide versatility of materials and substrates choice and high deposition rates to process an object in a reasonable period of time. Avoiding multi-step processing according to the required materials and the size of the patterns is also a major advantage. Among the foreseen candidates, atmospheric 
pressure micro-plasma treatments can be envisaged in a wide range of applications from electronic to medicine [Mariotti (JPD 2011)]. Working under atmospheric conditions without resorting to low-pressure system reduces notably heavy infrastructure and manufacturing costs. Also, deposition at atmospheric pressure prevents damages to desorbing and temperature-sensitive substrates such as living materials. Over the last few years only, isolated nanometric objects as well as localized functionalization of surfaces were envisaged [Belmonte (JPD 2011), Belmonte (JTST 2011), Motrescu 2012]. Perspectives on atmospheric pressure plasmas for nanofabrication have the distinction of being diverse and very exciting [Mariotti, JPD 2011]. For example, micro-optical waveguides [Hollander (2003)], vias-filling in electronic engineering or fabrication of integrated circuits in the microelectronic industry are subjected to intensive miniaturization. Both precise etching and deposition that are required in integrated manufacturing have been achieved thanks to the development of new advanced plasma tools [Mariotti (JPD 2011)]. Henceforth, quasi one-dimensional materials can be reached using laterally confined deposition by means of micro-sized injectors. In the early 2000s, Holländer and Abhinandan [Hollander (2003), Abhinandan (2004)] deposited localized quartz-like and hydrocarbons spots and lines from hexamethyldisiloxane (HMDSO) and acetylene respectively under vacuum. They used a stainless steel capillary of $250 \mu \mathrm{m}$ (inner diameter) of as injector. Once introduced in the vacuum chamber (0.2-2 mbar), the gas jet flows through the Radio-Frequency (RF) or Microwave (MW) excitation zone. The peak height of the needle-like coatings grown from $\mathrm{C}_{2} \mathrm{H}_{2}$ could reach $\sim 2.4 \mathrm{~mm}$ and the width at the half of the maximum height was $440 \mu \mathrm{m}$. Deposition experiments can be considered as simple decomposition reaction of a gaseous precursor assisted by atmospheric pressure plasma. In addition, APPJs demonstrate that nanometric patterns (lines or even more complex shapes) can be etched on silicon substrates with a good directionality, using noble gas plasma jet injected by means of a nano-capillary [Kakei (2010)]. These results demonstrate also that 
nano-sized charged particles can be taken out from the plasma jet with nano-capillary. In this way, both deposition (“writing”) and etching (“erasing”) modes can be coupled and embedded in the same material printing device by means of various suitable capillaries used as localized precursor or plasma jet sources. This novel approach based on APPJs allows any end-user to "write/erase" patterns over a surface without resorting to specific and expensive masks. In addition, tridimensional printing can be easily solved by using a moving XYZ stage where the gap between the capillary and the substrate surface can be continuously adjusted. Furthermore, in order to limit the use of harmful and flammable products in the semiconductor industry such as silane, tetraethoxysilane, trimethylaluminium...), localized treatments with small quantities of precursors and high conversion rates reduce the generation of hazardous contaminants and avoids fluorinated gases emission that promotes the greenhouse effect.

Synthesizing localized a-C:H coatings is an ideal case study for miniaturization since needle-like deposits were obtained with $\mathrm{C}_{2} \mathrm{H}_{2}$ as precursor in a recent past. This achievement showed that a-C:H polymers exhibit very interesting tribological properties. Localized a-C:H coatings are good candidates for low frictional coatings in solid lubrication applications and can be integrated onto stressed moving parts in micro-mechanical systems [Hollander (2003), Shimizu (2003)]. Indeed, a-C:H have extremely low friction coefficients ranging between $\mu=0.05$ [Miyake (1987)] and 0.35 [Zaidi (1994)] depending on the $\mathrm{H}$ content. a-C:H films are subjected to intensive research in this objective [Fontaine (2001), Comprehensive Hard Materials (2014)]. In addition, low density, high hardness, chemical inertness and infrared transparency make it an ideal material for protective optical coatings. According to the Robertson diagram [Robertson (1998), Robertson (2002)], hard a-C:H diamond like carbon (DLC) is better than soft a-C:H Polymer Like Carbon (PLC with $\mu<0.15$ ) for tribological applications. A progressive transition from soft to hard a-C:H is typically observed according 
to the hydrogen incorporation into the film. The friction and wear performances of a-C:H are significantly affected by the structural nature of the films $\left(\mathrm{sp}^{3} / \mathrm{sp}^{2}\right.$ hybridization, density, hydrogen content, surface energy, roughness...) and the surrounding conditions [Chen (2013)]. For example, the $\mathrm{sp}^{2} / \mathrm{sp}^{3}$ ratio is known to lead to soft or hard a-C:H materials depending on the hydrogen content in the structure. The experimental conditions can induce a domain transition from soft to hard a-C:H depending on the deposition parameters such as ion bombardment energy in the case of low-pressure deposition, gas composition or substrate temperature [Manage in thesis (1998), Manage (2000)]. Finally, the chemical characteristics of the deposited patterns under atmospheric conditions are a key point in the formation of a$\mathrm{C}: \mathrm{H}$ patterns and their subsequent mechanical properties. In this regard, the chemical control of the surrounding gas is essential.

Although APPJs are very attractive as it was mentioned before, the main drawback with APPJs is that plasmas cannot be reduced indefinitely in size. Indeed, the Debye length set a minimal size below which no ionized medium can be sustained. If a plasma is confined in a cavity comparable in size to the Debye length, shielding of the charge by the plasma and quasi-neutrality can no longer be preserved. Typical value of the Debye length for APPJs is between 10-100 $\mu \mathrm{m}$ [Eden (2006), Mariotti (JPD 2010)]. On that account, deposition of submicro-patterns using APPJs containing both the plasma gas and the precursor as it has been reported by Holländer and Abhinandan, becomes impossible below $10 \mu \mathrm{m}$ [Hollander (2003), Abhinandan (2004)]. In the objective of ultra-miniaturization of patterns, a convenient configuration has to be necessarily envisaged. This problematic has been dealt with using a novel approach by separating the precursor micro-jet from the plasma jet. The benefit is the possibility to work under post-discharge conditions to reduce significantly the temperature of the treated zone near the room temperature and to prevent damage of alterable substrates. The use of a moving stage using a controlled micrometric displacement can be done to realize 
localized spots, lines or more complex geometries over any substrate. This work emphasizes the opportunity of decreasing the size of a-C:H patterns from a micrometric to a nanometric scale. To determine optimal hydrodynamics conditions, a parametric study of the experimental setup scanning the main process parameters (capillary-plasma distance, capillary diameter, precursor pressure...) is proposed at the beginning of this paper. Throughout this article, we will show that the control of the chemical composition of the surrounding gas is an extremely important condition to reach ultrafine hydrocarbon patterns.

\section{Experimental details}

a-C:H micro and nano-patterns were deposited on polished intrinsic silicon $\{100\}$ oriented surfaces of $500 \mu \mathrm{m}$ in thickness. Prior to the treatment, substrates were successively cleaned in acetone and methanol, treated in an ultrasonic bath and put on the substrate stage for exposition to the argon plasma jet (see Figure 1). This plasma jet is generated thanks to a surfatron wave-launcher operating at atmospheric pressure. The plasma discharge is sustained by microwaves generated using a Sairem power generator operating at $2.45 \mathrm{GHz}$. The power absorbed by the plasma was fixed at $20 \mathrm{~W}$ for this work. To drive the afterglow on the silicon substrate, a curved fused silica tube of $3 \mathrm{~mm}$ in diameter was positioned inside the resonant cavity. The argon flow rate injected into the fused silica tube was fixed at $50 \mathrm{sccm}$ (standard cubic centimeter per minute). $\mathrm{C}_{2} \mathrm{H}_{2}$ (AAS27 type sold by Air Liquide) was used as gaseous chemical precursor. $\mathrm{C}_{2} \mathrm{H}_{2}$ is injected into an aluminosilicate glass capillary machined to control the shape and size of its extremity. Capillaries could be forged from $0.64 \mathrm{~mm}$ (initial inner diameter) down to $500 \mathrm{~nm}$ using a micropipette puller system (Sutter instruments) and a

microforge (WPI instruments). In this work, aluminosilicate glass was chosen for better 
performance in a high temperature range: it has a low thermal expansion coefficient $\left(4.10^{-6} \mathrm{~K}^{-}\right.$ ${ }^{1}$ ) and a higher softening point $(1170 \mathrm{~K})$ in comparison with borosilicate glass $(1090 \mathrm{~K})$. Consequently, aluminosilicate glass has a greater chemical durability and can withstand higher operating temperatures than borosilicate glass near the discharge region. The capillary is positioned with respect to the extremity of the apparent glow discharge, which can be defined approximatively as the end of the discharge. In a microwave discharge, the effective end of the discharge is the place where the electronic density is equal to the critical density which corresponds roughly to the end of the emissive zone.
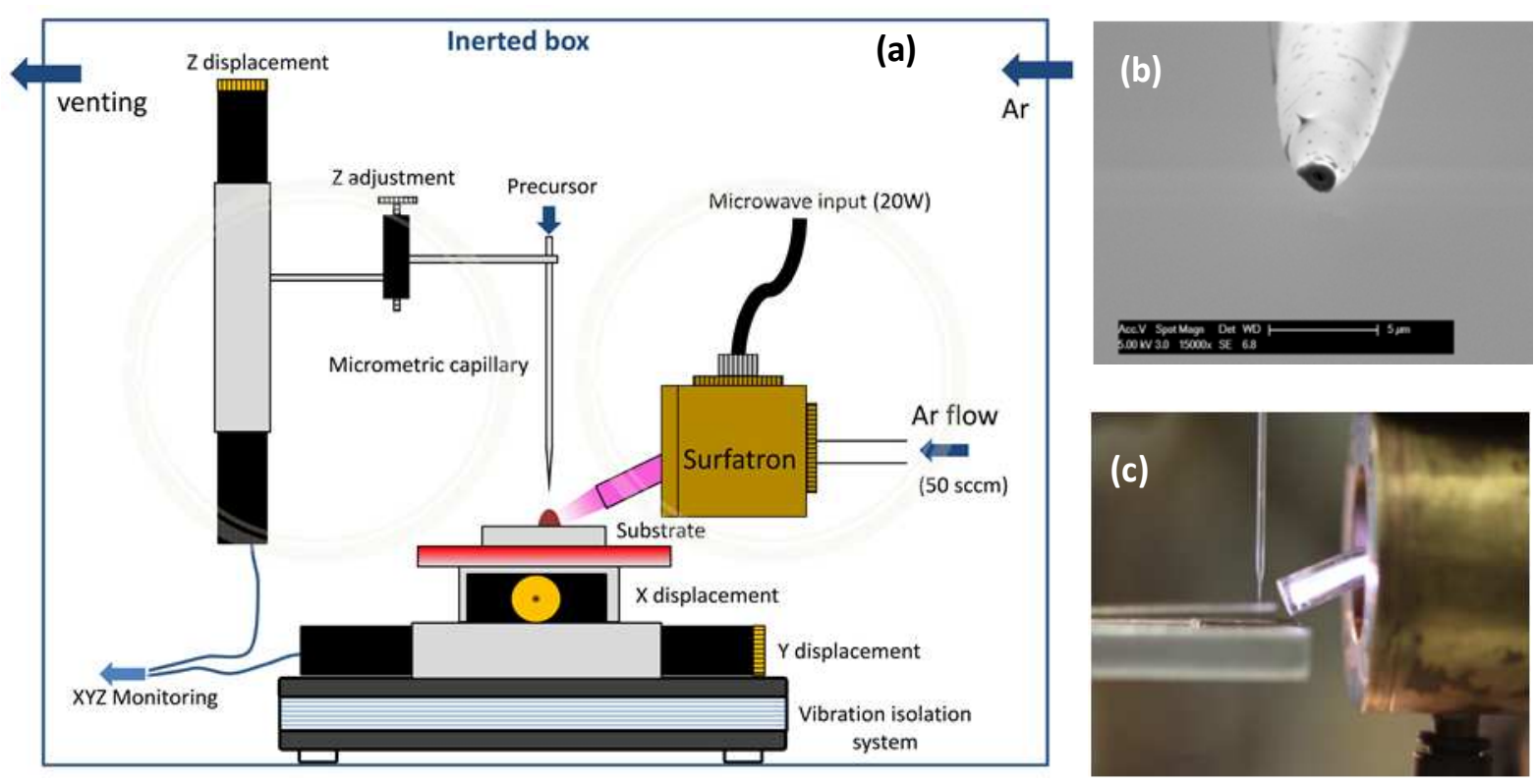

Figure 1. (a) Schematic representation of the experimental device operating at atmospheric pressure. (b) Extremity of a forged aluminosilicate glass capillary with an inner diameter of $500 \mathrm{~nm}$. (c) Picture of the treatment zone.

With the purpose of minimizing unwanted vibrations and improving the resolution of patterns, the experimental device was supported by an isolating table (Accurion Halcyonicsi4). The XY movement of the substrate is managed thanks to a linear XYZ stage while the $Z$ displacement is used to adjust precisely the capillary-substrate distance (linear stage LS-110 
PI Micos). The accuracy of the displacement is $\pm 0.1 \mu \mathrm{m}$. The Z-position between the capillary and the substrate surface was kept constant throughout this work (about $50 \mu \mathrm{m}$ ). The acetylene micro-jet flow rate depends on the inner capillary diameter and was regulated according to the $\mathrm{C}_{2} \mathrm{H}_{2}$ upstream pressure imposed in the capillary entrance thanks to the pressure regulator at the exit of the gas bottle. The minimal upstream pressure required to observe an acetylene jet is measured for different capillary diameters leading to specific hydrodynamic conditions, as discussed in the next section.

Bearing in mind that air moisture and oxygen possibly affect the chemistry of the a$\mathrm{C}: \mathrm{H}$ deposition at atmospheric pressure, we had to inert the treatment area. In addition, encapsulation of the experimental device prevents contamination of the user by possible nanoparticles and suppresses health damage. Isolation from the surrounding atmosphere is achieved by putting the experimental device in a Plexiglas box filled with argon (Alphagaz 1) provided by Air Liquide, with a maximum content in $\mathrm{H}_{2} \mathrm{O}$ and $\mathrm{O}_{2}$ below 3 ppm and 2 ppm respectively. The quality of argon inerting is controlled in real time by measuring the relative humidity $(\mathrm{Rh})$ and the oxygen content. This device allows us to rapidly reach $0.1 \% \mathrm{O}_{2}$ and $1 \% \mathrm{Rh}$ (less than 20 minutes). However, the inerting quality is probably better, these values corresponding to the oxygen and relative humidity sensitivities of the probes.

The morphology of a-C:H patterns was characterized by scanning electron microscopy (SEM) using a Phillips FEG-XL30S microscope and the topology was evaluated by Atomic Force Microscopy (AFM) using a Bruker Innova ${ }^{\circledR}$ microscope operating in tapping mode. The effect of the argon inerting rate on the post-discharge chemistry was analyzed by optical emission spectroscopy (OES) using a Jobin-Yvon TRIAX 550 spectrometer equipped with a $1200 \mathrm{gr} / \mathrm{mm}$ diffraction grating blazed at $500 \mathrm{~nm}$ and a Horiba Jobin-Yvon i-Spectrum Two iCCD detector. The composition of the deposited a-C:H patterns was analyzed by Fourier 
Transformer Infrared spectroscopy (FTIR) for different argon inerting values. A Cary 600 Fourier Transform spectrometer was used in the range of wavenumbers [4000 - 800] $\mathrm{cm}^{-1}$ and mapping an analyzed area of $25 \times 25 \mu \mathrm{m}^{2}$ in reflection mode thanks to a microscope.

\section{Results and discussion}

\section{A. Experimental considerations - parametric study}

To start with, the examination of hydrodynamic conditions related to the acetylene jet flow is a useful step to check the applicability of the present decoupled approach. Indeed, for a given upstream pressure, the reduction of the capillary diameter dramatically affects the pressure inside the capillary and, consequently, the acetylene flow rate as well as the deposition rate. The relative acetylene upstream pressure in the capillary (with regard to the atmospheric pressure) was measured using a Bourdon gauge. The relative upstream pressure was determined by dipping the capillary in ethanol. The emergence of a stream of bubbles indicates the minimum of the upstream pressure to impose. The relevant hydrodynamic parameters $\left(\mathrm{C}_{2} \mathrm{H}_{2}\right.$ flow rate, jet velocity and Reynolds number) are calculated from the measured minimum relative upstream pressure (more details are given as supplemental material). Results are presented in figure 2: 

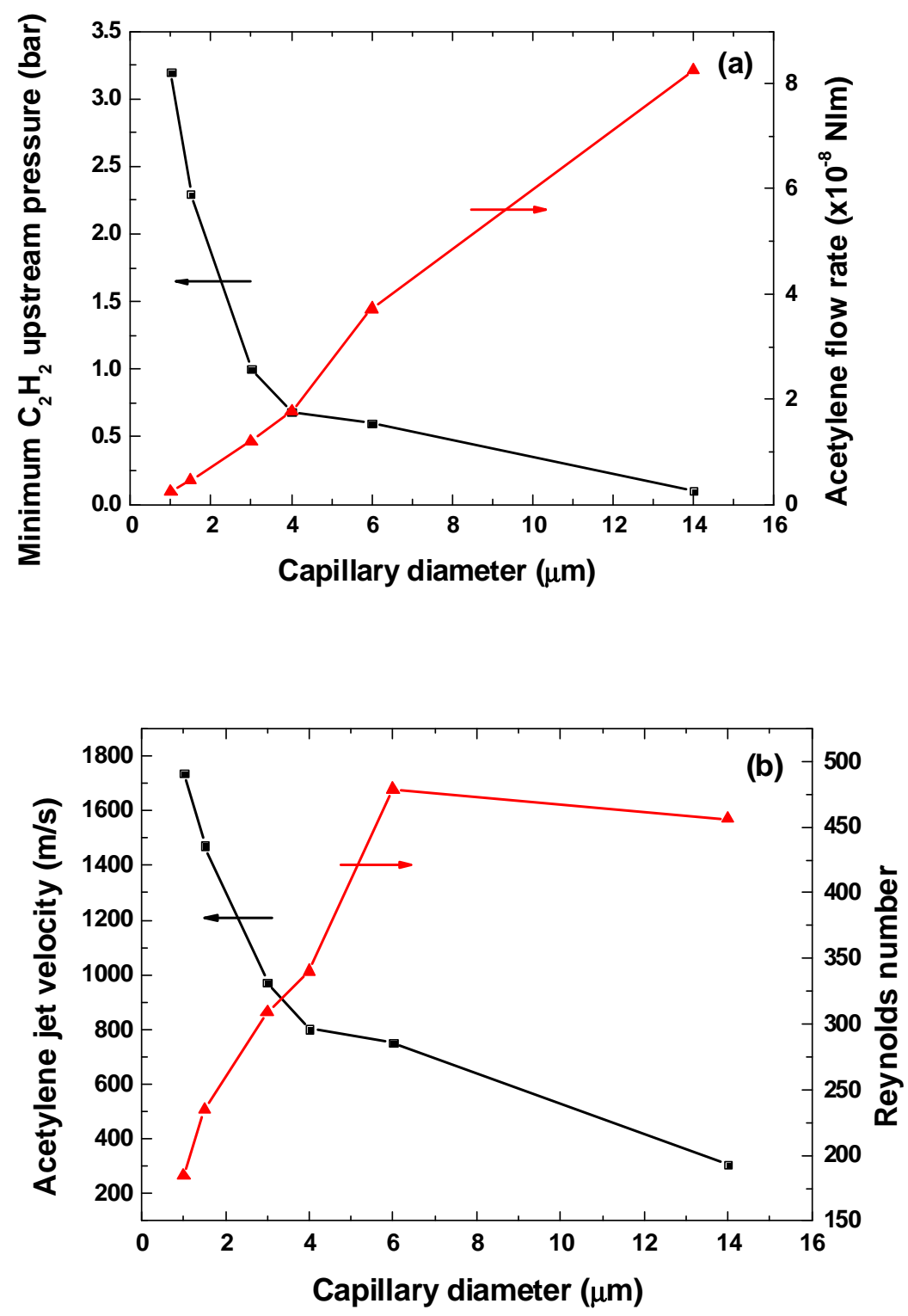

Figure 2. (a) Plot of the measured minimum $\mathrm{C}_{2} \mathrm{H}_{2}$ upstream pressure and the corresponding acetylene flow rate for different capillary diameters. (b) Evolution of the calculated hydrodynamic parameters (acetylene jet velocity and Reynolds number) as a function of the capillary radius, using the measured upstream pressure.

The measure of the relative minimum upstream pressure shows that the acetylene pressure in the capillary increases exponentially when the inner diameter decreases. The extrapolation of this curve to lower diameters demonstrates the possibility of getting nano-jets with an adapted pressure regulator system: e.g. the upstream pressure should be around 5 bars for a 
capillary of $200 \mathrm{~nm}$. The acetylene flow rate (considering the upstream pressure value) decreases linearly when the diameter decreases. The acetylene jet velocity can be estimated using the relation given in the supplemental material. Below $10 \mu \mathrm{m}$, the jet velocity exceeds the sound velocity $\left(580 \mathrm{~m} . \mathrm{s}^{-1}\right)$ taken at the post-discharge temperature (about $450 \mathrm{~K}$ ). For smaller diameters, the $\mathrm{C}_{2} \mathrm{H}_{2}$ jet reaches rapidly supersonic velocities (up to $1800 \mathrm{~m} . \mathrm{s}^{-1}$ for $\sim 1$ $\mu \mathrm{m}$ diameter). Then, the residence of the precursor in the post-discharge region becomes extremely short. For example, if the distance from the capillary to the substrate is, say, $50 \mu \mathrm{m}$, the species residence time is $1.0 \times 10^{-7}$ and $2.9 \times 10^{-8} \mathrm{~s}$ for capillary diameters of 10 and $1 \mu \mathrm{m}$ respectively. The drastic diminution of the residence time necessarily leads to a weaker consumption of acetylene for thinner capillaries. On the other hand, the decrease in the flow rate combined to the reduction of the exit section of the jet should certainly promote the reaction of $\mathrm{C}_{2} \mathrm{H}_{2}$ with active surrounding species transported to the post-discharge. More specifically, the penetration length (compared with the total section of the jet) of the active species by gaseous diffusion in the acetylene jet is greater when its diameter is reduced. This typical diffusion length in which the acetylene decomposition takes place can be regarded as a skin effect. As a result, the conversion rate of acetylene and the reaction of its products with active species reaching the post-discharge and the surrounding medium are probably widely enhanced for thinner capillaries.

In fluidics, the flow regime of a gas is usually defined by the Reynolds number. The Reynolds number computed from acetylene velocities and flow rates remains lower than 500 whatever the capillary diameter (more details are given in supplemental material). If we assume that the transition from a laminar to a turbulent regime occurs around 2000 [Seo (2010)], these values indicate that the acetylene jet is laminar, which ensures the stability of the flow and consequently the localization of precursor onto the surface. 
Another crucial parameter is the position of the acetylene jet in the post-discharge region. The distance between the capillary and the extremity of the glow discharge is very important, if we consider that the decay of the concentration of active species in the flowing afterglow is sufficiently fast to significantly affect the dissociation rate of the precursor. Consequently, a non-negligible dependence of the deposition rate on the position of the capillary in the postdischarge region can be expected. Figure 3 depicts the evolution of the deposition rate of a$\mathrm{C}: \mathrm{H}$ spots realized in static condition versus the capillary-plasma distance:

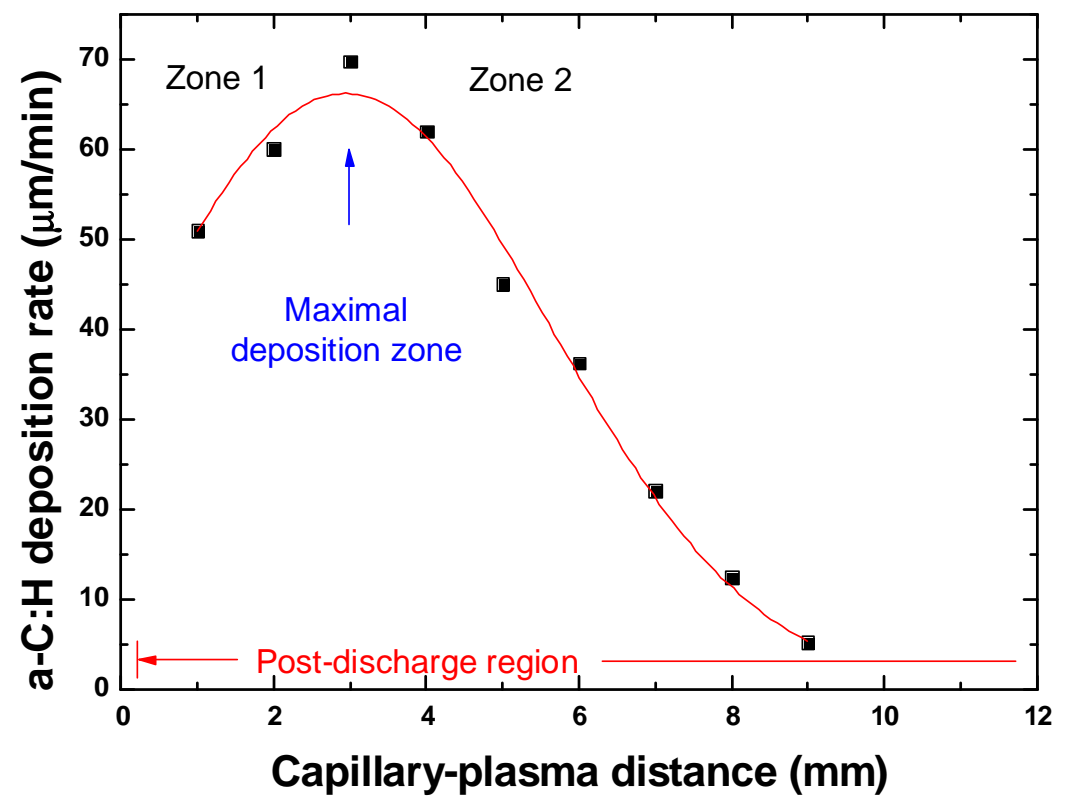

Figure 3. Evolution of the growth rate of a-C:H spots versus the distance in afterglow, defined as the distance between the capillary ( $5 \mu \mathrm{m}$ in inner diameter) and the end of the discharge.

The evolution of the deposition rate versus the plasma distance can provide several hints about the implicated species and an approximate scheme of growth mechanisms. This profile can be explained by means of active species distribution involved in the acetylene decomposition. Different reactive species could be identified in the plasma glow area: atomic 
oxygen $(\mathrm{O})$, hydroxyl $(\mathrm{OH})$, atomic hydrogen $(\mathrm{H})$ and nitric oxide $(\mathrm{NO})$. The presence of these different species was determined by OES measurements and will be discussed in detail in part C. Ozone, which is a very labile species, is assumed to be weakly concentrated, because of the high temperature of the microwave plasma (a few hundreds of ${ }^{\circ} \mathrm{C}$ typically). Two deposition regimes are discernible when the capillary is moved away from the end of the glow discharge. Near the discharge (in zone 1), the deposition rate increases up to a maximum located $3 \mathrm{~mm}$ downstream the plasma. It is useful to give an estimation of the required time of species coming from the plasma to travel this distance. Keeping an argon flow rate of $50 \mathrm{sccm}$ and an inner diameter of $3 \mathrm{~mm}$, the velocity of the plasma jet is close to $0.18 \mathrm{~m} \cdot \mathrm{s}^{-1}$. The maximum located at $3 \mathrm{~mm}$ is reached at $0.15 \mathrm{~ms}$. This time is typical of a chemical reaction associated to the activation of a precursor coming from the plasma. It is known that the main precursor involved in the hydrocarbon film growth is $\mathrm{C}_{2} \mathrm{H}_{2}{ }^{+} \cdot \mathrm{C}_{2} \mathrm{H}_{2}{ }^{+}$is mainly products by $\mathrm{Ar}^{+}$ ions and gives $\mathrm{CH}, \mathrm{C}_{2} \mathrm{H}$ and $\mathrm{C}_{2}$ as secondary by-products [Fauchais (1997), Asmussen (2002), Benedikt (2002), Pothiraja (2012)]. Since the recombination of $\mathrm{Ar}^{+}$is very fast, his transport in the post-discharge region is limited and cannot lead to the direct formation of $\mathrm{C}_{2} \mathrm{H}_{2}{ }^{+}$. Then, the presence of $\mathrm{Ar}^{+}$ions can be ruled out in the dissociation mechanisms of $\mathrm{C}_{2} \mathrm{H}_{2}$. However, the lifetime of metastable argon Ar* in the post-discharge is longer [Bussiahn (2010), Yu (2006), Xiong (2010)]. The density of neutral argon atoms and $\mathrm{C}_{2} \mathrm{H}_{2}$ molecules is close to $\sim 10^{19} \mathrm{~cm}^{-3}$, whereas the density of Ar* is lower by about 7 orders of magnitude $\left(\sim 10^{12} \mathrm{~cm}^{-3}\right)$. In atmospheric condition, $\mathrm{O}_{2}$ and $\mathrm{H}_{2} \mathrm{O}$ molecules are present at fairly high concentration in the post-discharge by gaseous diffusion (their density is about $\sim 10^{15} \mathrm{~cm}^{-3}$ ). Consequently, Ar* is rapidly quenched by $\mathrm{H}_{2} \mathrm{O}$ and $\mathrm{O}_{2}$ to generate $\mathrm{OH}$ and $\mathrm{O}$ as active species [Leblond (1981)]. The time required for the chemical activation of $\mathrm{OH}$ and $\mathrm{O}$ is typically of $\sim 10 \mathrm{~ms}$. In this way, the increase in the deposition rate in the zone 1 up to $3 \mathrm{~mm}$ is strongly correlated with the formation of $\mathrm{OH}$ and $\mathrm{O}$ precursors. Next, $\mathrm{OH}$ and $\mathrm{O}$ are able to reach the deposition zone by 
diffusion and react directly with $\mathrm{C}_{2} \mathrm{H}_{2}$ to form $\mathrm{C}_{2} \mathrm{H}$ and regenerate $\mathrm{H}_{2} \mathrm{O}$ or $\mathrm{OH}$ respectively. Thanks to this mechanism, the lateral growth rate of the spots reaches a maximum at $3 \mathrm{~mm}$ downstream the discharge. In zone 2, a rapid decrease in the growth rate occurs up to $10 \mathrm{~mm}$ where deposition becomes negligible. This decrease is attributed to the depletion of the active species $\mathrm{O}$ and $\mathrm{OH}$ since the concentration of $\mathrm{C}_{2} \mathrm{H}_{2}$ is constant all along the profile.

Finally, the optimal zone for the deposition is found at $3 \mathrm{~mm}$, at the transition between zone 1 and zone 2 . Subsequently, the capillary was placed at this transition position for all the results presented hereinafter.

\section{B. Deposition under atmospheric environment}

Hydrocarbon spots were deposited by means of different capillary sizes $(2,1$ and 0.5 $\mu \mathrm{m}$ in inner diameter) without inerting by argon. The deposition time was set at 1 second in static condition. The deposition time was kept constant for this series. SEM micrographs of the spots are presented in figure 4 for each capillary: 


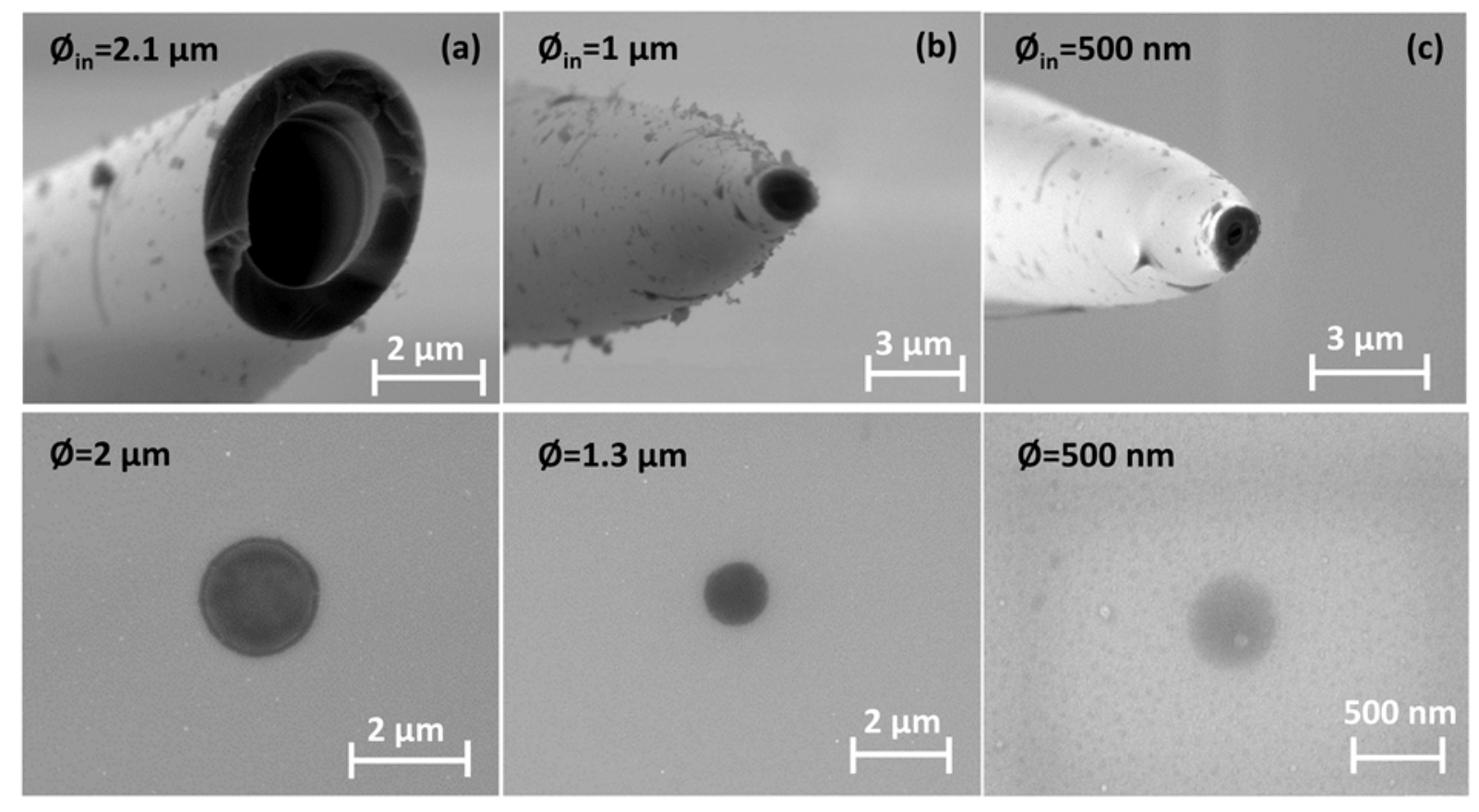

Figure 4. SEM micrographs of glass capillaries (inner diameters: $2 \mu \mathrm{m}$ (a), $1 \mu \mathrm{m}$ (b) and 0.5 $\mu \mathrm{m}(\mathrm{c}))$ and the corresponding hydrocarbon spots deposited using the capillaries showed above.

SEM micrographs of patterns show that hydrocarbon spots have a regular circular shape and that spots are well localized on the substrate surface, where the capillary is positioned. The comparison of spots obtained by reducing the glass capillary size reveals a very good congruence between the inner diameter of the capillary and the diameter of the spot. However, our experimental device does not allow the use of capillaries below $500 \mathrm{~nm}$ in diameter because the acetylene pressure regulator is limited to 4 bars. As described previously, the acetylene upstream pressure in the capillary increases exponentially (see figure 2a) and the maximum upstream pressure value is reached for a diameter of $500 \mathrm{~nm}$.

It is worth noting that neither spreading of hydrocarbon contamination nor soiling area are observed around the spots. This observation means that deposition from the acetylene jet is very directional while the broadening of the spot should be still observed considering its sprawl over the silicon surface. We suppose that the localization is enhanced by the etching of 
carbon induced by the uptake of a small amount of oxygen and water in the acetylene jet by gaseous diffusion. $\mathrm{O}_{2}$ or $\mathrm{H}_{2} \mathrm{O}$ species are in high concentration in the non-inerted surrounding atmosphere. As described above, the incorporation of $\mathrm{H}_{2} \mathrm{O}$ by diffusion in the immediate vicinity of the $\mathrm{C}_{2} \mathrm{H}_{2}$ jet inhibits the growth of a-C:H by reaction with $\mathrm{C}_{2} \mathrm{H}$ which is the main species involved in the growth of a-C:H patterns. Consequently, the limitation of acetylene decomposition around the acetylene jet when it spreads over the surface improves localization. In addition, it is likely that atomic oxygen and $\mathrm{OH}$ radicals etch the a-C:H spot around the deposited pattern [Jeong (1998), Jeong (1999)]. Although O has a relative short lifetime at atmospheric pressure compared to NO for example [Seo (2010)], atomic oxygen can reach the $\mathrm{C}_{2} \mathrm{H}_{2}$ jet and the deposition area provided that the plasma jet velocity is sufficient. The lifetime of atomic oxygen is in the range of hundreds of milliseconds [Lu (2008)], [Ono (2000)]. An assessment of the moving distance O can be made from the argon flow rate $(50 \mathrm{sccm})$. The velocity of plasma jet is close to $0.18 \mathrm{~m} \cdot \mathrm{s}^{-1}$ and the distance over which $\mathrm{O}$ can be transported is in the range $20-100 \mathrm{~mm}$. Then, the presence of $\mathrm{O}$ is possible at a sufficiently high concentration to permit the etching of the hydrocarbon pattern in zones 1 and 2, thus enhancing localization.

As a conclusion, we can expect that $\mathrm{O}$ and $\mathrm{OH}$ act both as activators for the deposition of a-C:Hand as etching agents around the deposited spots.

However, when deposition of non zero-dimensional patterns was tried, the role of these reactive oxygen-containing species becomes more complex. Indeed, hydrocarbon lines were deposited in dynamic mode by setting the substrate velocity at $10 \mu \mathrm{m} . \mathrm{s}^{-1}$. We observed that a rapid and progressive dewetting of the deposited lines occurs when the samples are taken out of the plasma, leading to the formation of aligned micro-droplets. In Figure 5, FTIR spectra of two large a-C:H(:O) spots (about $50 \mu \mathrm{m}$ in diameter) are depicted. These coatings 
were deposited in the same conditions and under atmospheric environment $(30 \% \mathrm{Rh}$ and $20 \% \mathrm{O}_{2}$ ). FTIR spectra were recorded just after treatment and after 1 day to evaluate the ageing of the sample.

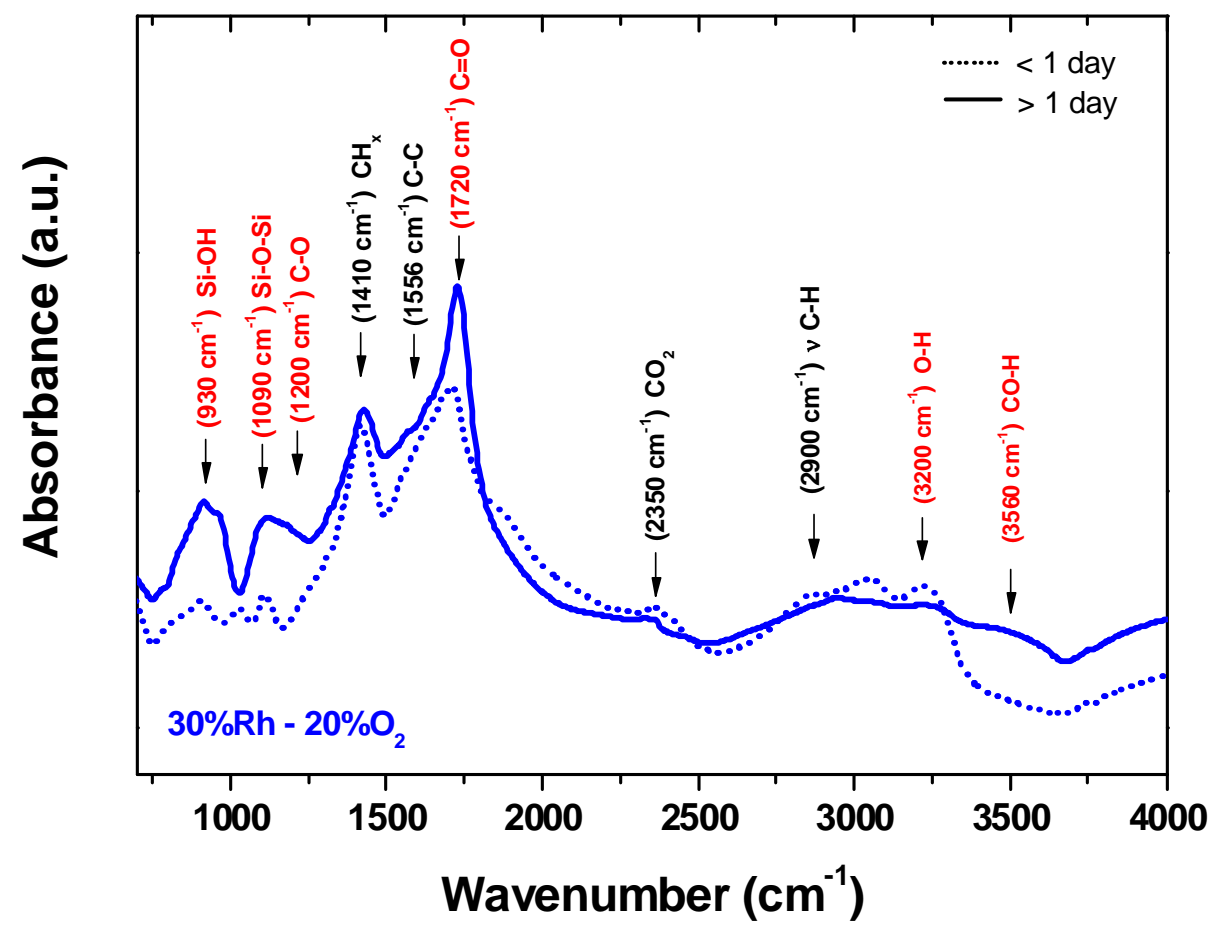

Figure 5. FTIR spectra of two a-C:H:O spots (about $50 \mu \mathrm{m}$ in diameter) deposited under atmospheric conditions just after treatment (a few minutes) and after 1 day.

First, the increase in the peak intensity located at $1080 \mathrm{~cm}^{-1}$ and assigned to the $\mathrm{Si}-\mathrm{O}-$ Si stretching mode [Luna-Lopez (2009)] is due to the disclosing of the apparent surface of the native silica layer after dewetting of the deposited pattern. The comparison of both spectra indicates that the ageing of patterns shows a dramatic increase in bonds between carbon and oxygen-containing groups (mainly carbonyl and carboxyl groups) whereas no significant evolution is observed with hydrogenated carbon groups. A significant increase in the intensity of two absorption bands attributed to $-\mathrm{C}=\mathrm{O}$ groups (carbonyl stretching mode in ketone groups) and CO-H groups (stretching mode) located at 1720 and $3560 \mathrm{~cm}^{-1}$ respectively, is 
clear after 1 day [Manage (2000), Konshina (1997), Marcinauskas (JOAM 2010), Mawhinney (2001)]. This enlargement is also accompanied by an enhancement of the absorption band centered at $1200 \mathrm{~cm}^{-1}$, which is assigned to the stretching modes of the $\mathrm{C}-\mathrm{O}$ surface moieties [Fanning (1993), Mawhinney (2001)]. The presence of the broad band arising in the vicinity of $3270 \mathrm{~cm}^{-1}$ comes from hydrogen bonded in O-H hydroxyl groups [Konshina (1997), Manage (2000)]. This signal decreases slightly after 1 day but not enough to reveal a chemical consumption of hydroxyl groups. The absorption peak around $\sim 930 \mathrm{~cm}^{-1}$ is the signature of the $\mathrm{Si}-\mathrm{OH}$ groups on the apparent substrate after dewetting [Theil (1990)]. The presence of this strong absorption line shows the activation and the modification of the silicon surface by the afterglow discharge. It should be mentioned that the dewetting process starts immediately after deposition. Hence, the absorption differences observed between a non-withdrawn sample from the post-discharge and after 1 day must be much larger in reality but these two spectra recorded after deposition are very representative of the reactivity of the deposited patterns. Considering the strong increase in oxygenated groups, it is therefore correct to say that the chemical composition of the coatings deposited under atmospheric environment is strongly affected by ageing in air.

The region $\sim 2900 \mathrm{~cm}^{-1}$ is assigned to numerous $\mathrm{C}-\mathrm{H}$ stretching modes corresponding to aliphatic groups. More specifically, this region can be divided into three bands related to $\mathrm{CH}_{3-2}$ symmetric and asymmetric stretching modes. The band at $2860 \mathrm{~cm}^{-1}$ represents $\mathrm{sp}^{3}$ $\mathrm{CH}_{2}$ symmetric vibrations and the other two vibrations at around 2930 and $2960 \mathrm{~cm}^{-1}$ are assigned to $\mathrm{sp}^{3} \mathrm{CH}_{2}$ and $\mathrm{sp}^{3} \mathrm{CH}_{3}$ asymmetric stretching modes respectively [Liu (1997), Kulisch (2006), Marcinauskas, JOAM (2010)]. These vibrational modes do not appear to be significantly affected by the ageing. 
The identification of absorption bands observed at $\sim 932$ and $\sim 3046 \mathrm{~cm}^{-1}$ is less straightforward and can be debatable. We reckon that the absorption peak observed before 1 day at $\sim 3046 \mathrm{~cm}^{-1}$ could result from the stretching mode of $\mathrm{sp}^{2} \mathrm{C}-\mathrm{H}$ aromatic groups. This mode is also observed at wavenumbers around $\sim 3060 \mathrm{~cm}^{-1}$ in the literature [Robertson (1991), Liu (1997), Mawhinney (2001)]. This assignment is justified by the presence of the aromatic $\mathrm{sp}^{2} \mathrm{C}-\mathrm{C}$ stretching mode at $1556 \mathrm{~cm}^{-1}$, also observed as a shoulder of the $\mathrm{C}=\mathrm{O}$ sharp peak at $1720 \mathrm{~cm}^{-1}$ and already found at $1583 \mathrm{~cm}^{-1}$ in the work by Robertson [Robertson (1991)]. The absorption broad peak appearing at $932 \mathrm{~cm}^{-1}$ can be attributed to the bending mode of C-H observed at $970 \mathrm{~cm}^{-1}$ by Salonen et al. [Salonen (2004)].

We assume that the conceivable mechanism of dewetting consists in the fast reaction of the $\mathrm{C}-\mathrm{O}$ surface moieties, carbonyl and hydroxyl groups with the atmospheric moisture when the sample is taken out of the post-discharge region [Smith (1995)]. This assumption was tested by heating a dewetted sample up to $670 \mathrm{~K}$ under a confocal microscope equipped with a heating stage. The rewetting of film occurs at a temperature of about $380 \mathrm{~K}$ and can be attributed to the thermal decomposition of the carboxylic acid functional groups under heating by dehydration $\left(\mathrm{H}_{2} \mathrm{O}\right.$ elimination $)$ and decarboxylation $\left(\mathrm{CO}_{2}\right.$ elimination in air) [Nguyen (JPC 1995), Valor (2002)]. The mechanism of the direct formation of carboxylic groups by direct ozonolysis of unsaturated carbon bonds must be regarded thoroughly since it was shown to be important by Mawhinney et al. [Mawhinney (2001)]. The ozonolysis reaction of unsaturated bonds such as alkenes to form very instable intermediate products, which decompose rapidly in carboxylic and carbonyl identities, is highly suspected to occur in a-C:H films exposed to $\mathrm{O}_{3}$ [Criegee (1975), Neeb (1996), Neeb (1998), Manoilova (1998)]. The reaction of ozone in the carboxylation reaction follows the ozonolysis mechanism: 


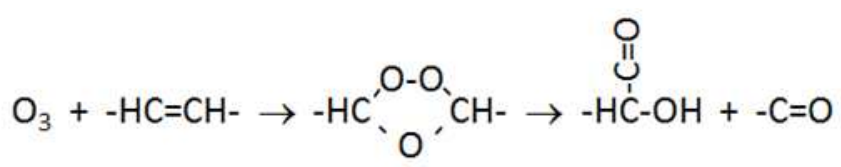

As indicated earlier, $\mathrm{O}_{3}$ concentration increases with the decrease in atomic oxygen concentration and temperature in the post-discharge region [Jeong (1998)]. However, the temperature measured in the deposition area reaches $450 \mathrm{~K}$. So, the temperature is still too high to allow the stability of $\mathrm{O}_{3}$. At this temperature, the ozone concentration must be very low because ozone is destabilized very rapidly as soon as the temperature rises above $380 \mathrm{~K}$. Deposition at high temperatures can lead to a spontaneous dewetting of a-C:H:O films. The dewetting of patterns arises after withdrawal of the samples from the thermal deposition area, which suggests a relaxation of thermal stresses. However, a-C:H(:O) patterns deposited under complete argon inerting at similar and even higher temperatures are not subjected to dewetting. This observation allows us to discard a purely thermal effect occurring on the chemical or mechanical stability of a-C:H:(O) patterns. Then, the direct reaction of free radicals $\mathrm{R} \cdot$ embedded in the a-C:H matrix with molecular oxygen coming from the atmosphere by gaseous diffusion should be strongly considered. This reaction can be described according to the following mechanism suggested by Bilek et al. [Bilek (2011), [Kosobrodova (2012), Kosobrodova (2014)]:

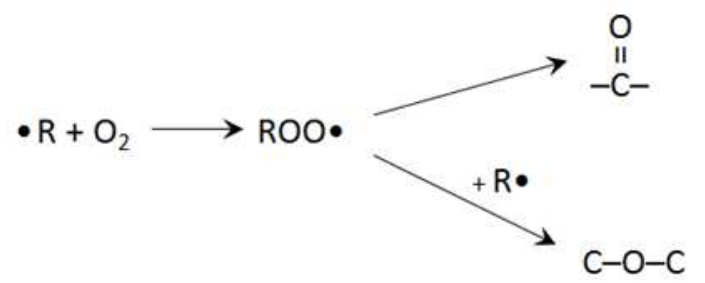




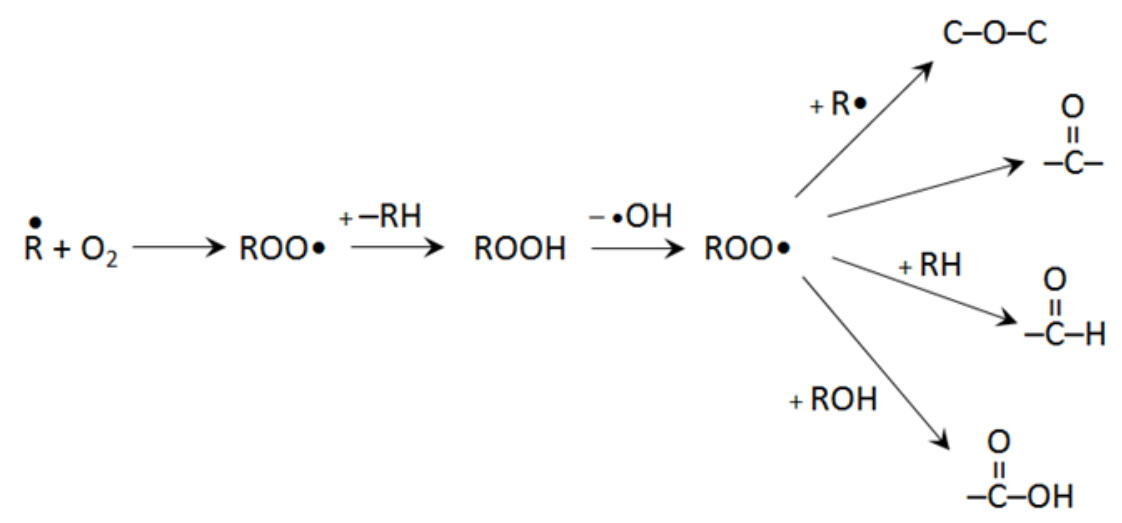

The direct oxidation of the dangling bonds by diffusion of molecular oxygen, leads mainly to the formation of $\mathrm{C}-\mathrm{O}-\mathrm{C}$ and $\mathrm{C}=\mathrm{O}$ groups that are highly subjected to the subsequent hydrolysis. This first mechanism can be accompanied by subsequent radical reactions that form $\mathrm{COH}, \mathrm{COOH}, \mathrm{C}=\mathrm{O}$ and $\mathrm{C}-\mathrm{O}-\mathrm{C}$ groups. This mechanism based on the reaction of dangling bonds is independent of the temperature and proportional to the $\mathrm{O}_{2}$ concentration in the atmosphere. Thus, oxidation of the carbon layer can be easily suppressed by inerting the surrounding medium, which was also confirmed in our work.

In summary, highly oxygenated and hydrogenated a-C:H:O patterns are subjected to strong subsequent chemical instabilities, which promotes contamination by atmospheric water and the destruction of coatings by dewetting. First, reaction of free radicals with molecular oxygen or water produces $\mathrm{C}-\mathrm{O}$ surface moieties and carbonyl groups in the post-discharge area. Then, dewetting by hydrolysis of these bonds is assisted by the ambient moisture. This affects significantly the structural and mechanical properties of the coatings. However, the subsequent dewetting promotes the localization of circular shaped a-C:H:O patterns. In case of more complex shapes deposited in dynamic condition, the composition of the atmospheric surrounding medium has to be carefully controlled to ensure both the chemical purity and stability (a-C:H instead of a-C:H:O) of patterns and to avoid dewetting. The last part of this paper concentrates on the effect of argon inerting. 


\section{Benefit of argon inerting on the chemical and structural stabilities of hydrocarbon patterns}

In order to minimize oxidation and subsequent dewetting of a-C:H patterns, the medium surrounding the deposition area was inerted by an argon flow injected into the Plexiglas box enclosing the experimental device (figure 1a). The evolutions of $\mathrm{O}_{2}$ and $\mathrm{H}_{2} \mathrm{O}$ partial pressures plotted against inerting time are presented in figure 6. To evaluate the effect of argon inerting on plasma chemistry and confirm the role of atomic oxygen and hydroxyl groups, characterizations were carried out by optical emission spectroscopy (OES) without acetylene injection. The optical fiber was positioned at the end of the plasma, by varying the flow rate of the inerting gas (Ar) down to the limit of detection $1 \% \mathrm{Rh}-0.1 \% \mathrm{O}_{2}$. In all spectra, hydroxyl radicals $\mathrm{OH}\left(306.0 \mathrm{~nm}: \mathrm{A}^{2} \Sigma^{+}, v=0 \rightarrow \mathrm{X}^{2} \Pi, v=0\right)$, atomic oxygen $\mathrm{O}(777.15 \mathrm{~nm}$ : triplet state), NH (336.0 nm: $\left.A^{3} \Pi^{+}, v=0 \rightarrow X^{3} \Sigma^{-}, v=0\right), N_{2}\left(337.10 n m: C^{3} \Pi_{u}, v=0 \rightarrow B^{3} \Pi_{g}, v=0\right), H(656.25$ $\mathrm{nm}: \mathrm{Ha}, n=3 \rightarrow 2)$ and NO (258.68 $\left.\mathrm{nm}: \mathrm{A}^{2} \Sigma^{+}, v=0 \rightarrow \mathrm{X}^{2} \Pi, v=3\right)$ transitions are clearly identified from tabulated data [Pearse (1976)]. For the sake of clarity, we have chosen to represent the intensity ratios of the transitions of detected species and that of the most intense argon line at $811.51 \mathrm{~nm}$. The evolutions of different ratios are reported in figure 7 as a function of the relative humidity $\mathrm{Rh}$. 


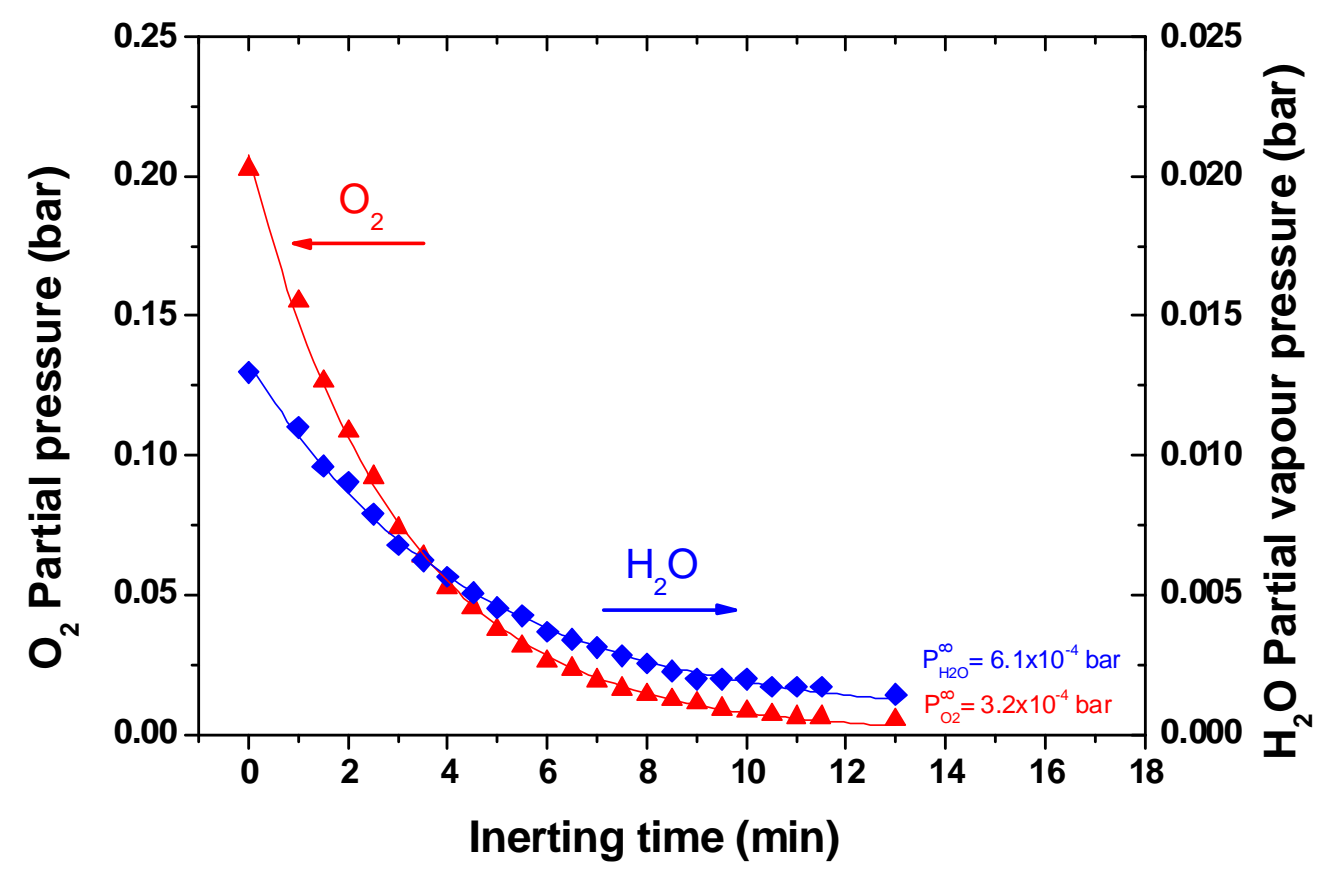

Figure 6. Evolutions of $\mathrm{O}_{2}$ and $\mathrm{H}_{2} \mathrm{O}$ partial pressures plotted against the time of box filling with argon.

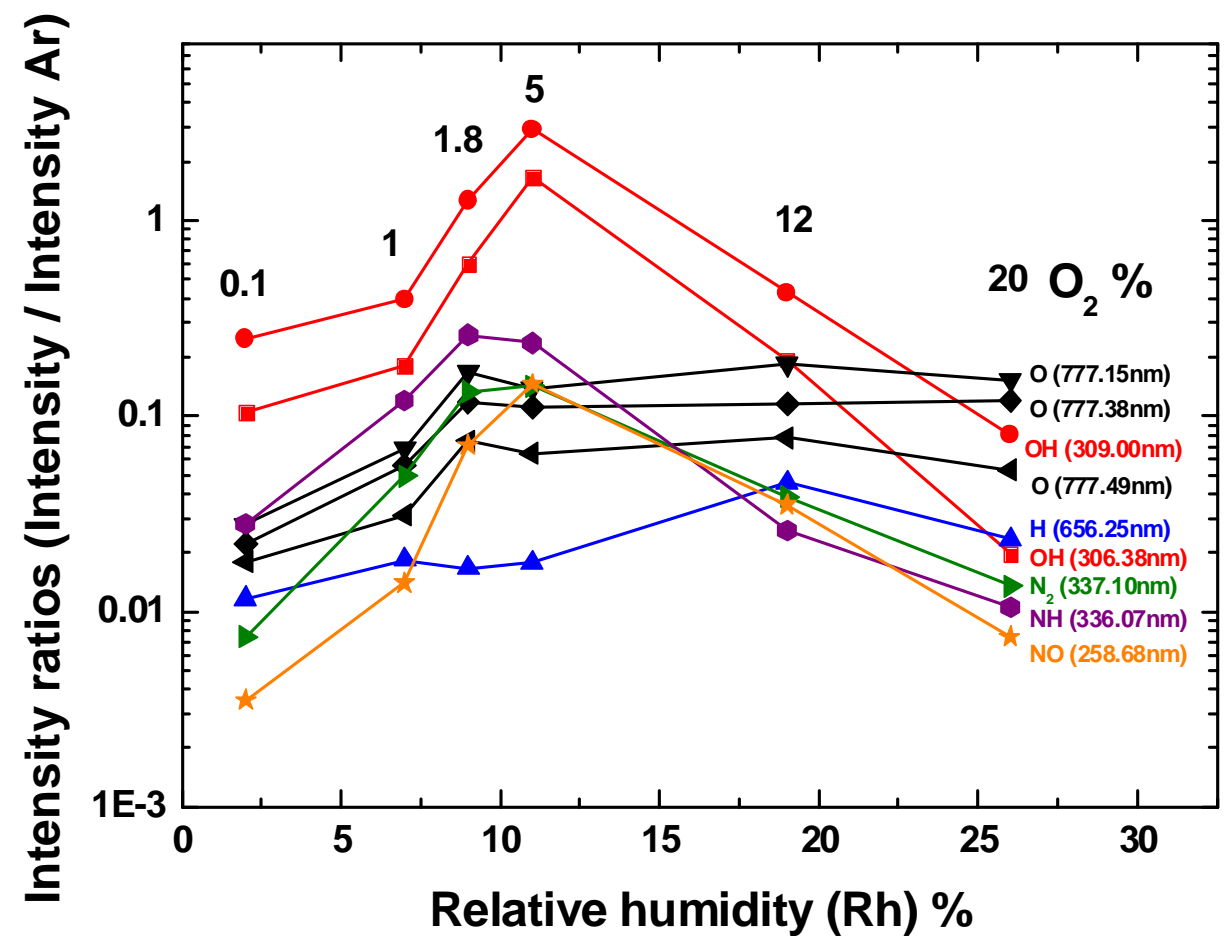


Figure 7. Intensity ratios of the transitions of main chemical impurities with respect to the most important argon transition at $811.51 \mathrm{~nm}$ as deduced from OES measurements at the end of the plasma.

Figure 6 shows that argon filling is accompanied by a drastic diminution of the $\mathrm{O}_{2}$ and $\mathrm{H}_{2} \mathrm{O}$ partial pressures after about $15 \mathrm{~min}$. The estimated maximal partial pressures by extrapolation of curves are $\sim 10^{-4}$ bar, i.e. $2.1 \% \mathrm{Rh}$ and $0.03 \% \mathrm{O}_{2} . \mathrm{O}_{2}$ and $\mathrm{H}_{2} \mathrm{O}$ concentrations are higher than the maximum impurities levels in argon announced at $5 \mathrm{ppm}$ and $3 \mathrm{ppm}$ respectively. OES measurements shown in figure 7 set out a clear picture on the inerting quality. It is interesting to focus on the atomic oxygen triplet transition at $777.15 \mathrm{~nm}$. The atomic oxygen concentration remains constant in the range $10-26 \% \mathrm{Rh}$ and $1.8-20 \% \mathrm{O}_{2}$. To explain this plateau, we have to consider two opposite effects. The presence of atomic oxygen lines in the post-discharge region are principally due to optical transitions of atomic oxygen formed in the discharge. Atomic oxygen has a very short lifetime and therefore oxygen lines appear as a very weak signal whereas the contribution of oxygen transitions in the optical spectrum prevails. When the flow rate of inerting gas increases, the intensity of oxygen transitions increases as the plasma expands out of the tube. In a discharge surface wave, this means that the critical density below which the plasma disappears is shifted downstream. Then, the electron density at a given position increases and so, the oxygen transition intensities if the concentration of oxygen is constant. However, the depletion of the oxygen content when the inerting argon flow rate increases balances out the increase in the electron density, and the intensity of oxygen transitions remains roughly constant between $10-26 \% \mathrm{Rh}$ and $1.8-20 \% \mathrm{O}_{2}$. Below $10 \% \mathrm{Rh}-1.8 \% \mathrm{O}_{2}$, the plasma extension stops, and the decrease in the intensity of the oxygen lines is due only to the improvement of inerting. OES measurements in the tube (results are not reported here) revealed that the concentrations of all contaminants 
are constant over the range of inerting argon flow rates as soon as the plasma tube is clean enough, which requires a few minutes after plasma ignition.

It is important to note that the $\mathrm{O}_{2}$ signals around $760 \mathrm{~nm}$ and $770 \mathrm{~nm}$ are associated with two emission bands of the oxygen atmospheric system $\left(\mathrm{b}^{1} \Sigma_{\mathrm{g}}^{+}, v=0\right) \rightarrow\left(\mathrm{X}^{3} \Sigma_{\mathrm{g}}^{-}, v=0\right)$ and $\left(\mathrm{b}^{1} \Sigma_{\mathrm{g}}{ }^{+}, v=1\right) \rightarrow\left(\mathrm{X}^{3} \Sigma_{\mathrm{g}}{ }^{-}, v=1\right)$ [Pearse (1976)] respectively are not reported because these lines are not detected in our spectra, unlike pure argon-oxygen plasmas. The lack of $\mathrm{O}_{2}$ can be related to the complete consumption of $\mathrm{O}_{2}$ diffused in the plasma to form atomic $\mathrm{O}$ and $\mathrm{NO}$. Metastable argon are directly involved in the reaction with $\mathrm{O}_{2}$ following the reaction $\mathrm{Ar}^{*}+\mathrm{O}_{2}$ $\rightarrow \mathrm{Ar}+2 \mathrm{O}$ described by Kutasi et al. [Kutasi (2011)]. Nevertheless, the lack of $\mathrm{O}_{2}$ lines in OES spectra along the inerting procedure does not mean that molecular oxygen is not present in the afterglow and its environment. Figure 6 shows that the $\mathrm{O}_{2}$ content is almost $10^{3}$ times lower after complete inerting while O lines intensities are only divided by 10 . Even though OES intensities are not directly representative of concentrations, a notable difference is observed between OES and inerting measurements. In complete inerting condition, we suppose that the plasma pumps probably residual $\mathrm{O}_{2}$ in the surrounding medium to form atomic $\mathrm{O}$. Thus, the area and the post-discharge are cleaned of $\mathrm{O}_{2}$ and the dewetting of the film is suppressed.

Lines attributed to $\mathrm{OH}$ radicals are detected in all $\mathrm{OES}$ spectra. The formation of $\mathrm{OH}$ radicals from $\mathrm{H}_{2} \mathrm{O}$ molecules can be done by ionized or metastable argon atoms. The increase in $\mathrm{OH}$ concentration while the box is filled with argon up to $12 \% \mathrm{Rh}-5 \% \mathrm{O}_{2}$, is due to the increase in the electron density, accompanied by an effective increase of $\mathrm{Ar}^{*}$ and $\mathrm{Ar}^{+}$species. Below $12 \% \mathrm{Rh}-5 \% \mathrm{O}_{2}, \mathrm{OH}$ radicals decrease by lack of water, which promotes the rise of argon metastable states. Finally, $\mathrm{OH}$ concentrations corresponding to low and high inerting degrees are quite similar and the expected $\mathrm{OH}$-free medium is not really observed although 
the $\% \mathrm{Rh}$ is 10 times lower after complete inerrting. On the other hand, it should be noted that if acetylene is introduced, the concentration of $\mathrm{OH}$ radicals must be much higher. Indeed, acetone is used as stabilization agent in acetylene storage and brings additional oxidizing species. The presence of $\mathrm{OH}$ and $\mathrm{O}$ in complete inerting conditions is not unwelcomed because these two species are involved in the dissociation of $\mathrm{C}_{2} \mathrm{H}_{2}$. Only molecular oxygen has to be suppressed to avoid the film oxidation and a subsequent dewetting. Also, it is more likely that depletion of $\mathrm{O}_{2}$ and $\mathrm{H}_{2} \mathrm{O}$, together with plasma extension, modify the dissociation mechanisms of $\mathrm{C}_{2} \mathrm{H}_{2}$. In a purified atmosphere, $\mathrm{Ar}^{*}$ generated in the plasma are able to attain more easily the $\mathrm{C}_{2} \mathrm{H}_{2}$ jet. The secondary chemical route consists most probably in the direct reaction of metastable argons generated in the plasma with $\mathrm{C}_{2} \mathrm{H}_{2}$ to form $\mathrm{C}_{2} \mathrm{H}$ as primary precursor involved in the a-C:H growth. With inerting and depletion of $\mathrm{O}_{2}$, oxidation of dangling bonds is completely suppressed in an Ar environment as it was observed. The depletion of $\mathrm{O}_{2}$ and the demise of dewetting support the idea that the proposed mechanism based on the oxidation by the atmospheric oxygen of radicals does operate.

Figure 8 depicts the FTIR absorbance spectra of large hydrocarbon spots deposited by varying the composition of the surrounding atmosphere. The FTIR spectra were acquired only a few minutes after deposition. 


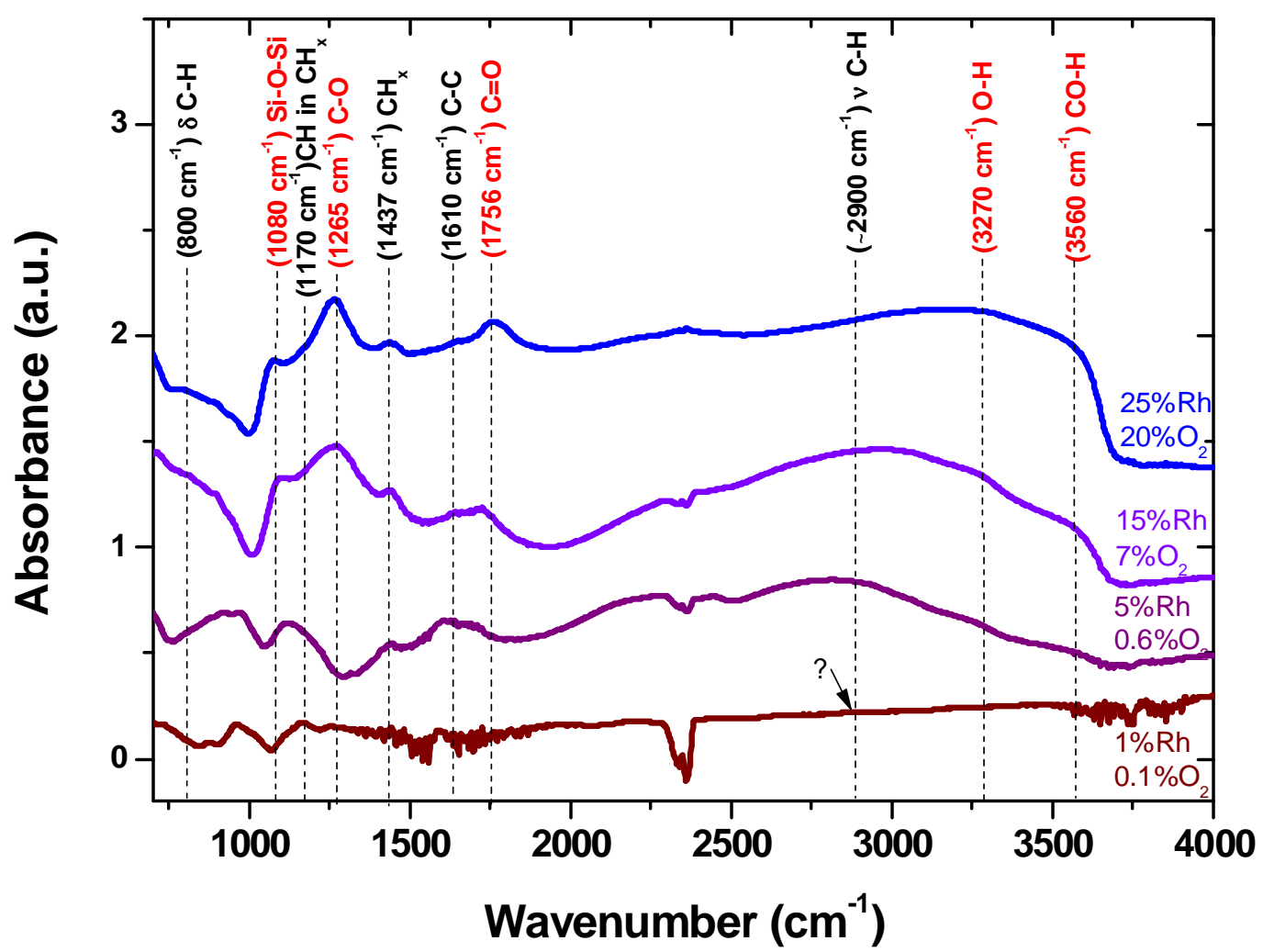

Figure 8. FTIR spectra of large a-C:H(:O) spots deposited by varying the inerting argon flow rate in the Plexiglas box (expressed in relative humidity $\mathrm{Rh}$ and oxygen content). All spectra were acquired a few minutes after deposition.

The influence of inerting on the optical properties of the deposited coating shows that absorption peaks corresponding to carbonyl and hydroxyl groups decrease dramatically as a whole. The $\mathrm{C}-\mathrm{O}, \mathrm{C}=\mathrm{O}, \mathrm{CO}-\mathrm{H}$ and $\mathrm{O}-\mathrm{H}$ groups localized at $1265,1756,3560$ and $3270 \mathrm{~cm}^{-1}$ progressively drops whereas no signal is observed at $1 \% \mathrm{Rh}-0.1 \% \mathrm{O}_{2}$. The occurrence of these peaks discloses the strong chemical reactivity of as-deposited coatings, whose hydrolysis is very fast in the air. All FTIR spectra were recorded at equivalent times after deposition and each spectrum is representative of the chemical stability of the a-C:H coatings after treatment. Besides, strong attenuation of the $\mathrm{Si}-\mathrm{O}-\mathrm{Si}$ absorption band located at $1080 \mathrm{~cm}^{-1}$ is observed when Ar inerting is improved. This attenuation comes from a weaker contribution of the dewetting process. The silicon surface covered by the a-C:H spots is better masked for higher 
inerting degrees. For that reason, inerting by argon ensures a higher chemical stability of the hydrocarbon patterns. In contrast, the hydrocarbon groups are entirely preserved up to $5 \% \mathrm{Rh}$ $0.6 \% \mathrm{O}_{2}: \mathrm{CH}_{\mathrm{x}}\left(1170 \mathrm{~cm}^{-1}\right), \mathrm{CH}$ bending $\left(1437 \mathrm{~cm}^{-1}\right)$ [Liu (1997)], olefin $\mathrm{sp}^{2} \mathrm{C}=\mathrm{C}$ stretching band (1610 $\mathrm{cm}^{-1}$ ) [Louh (2006), Marcinauskas Vacuum (2007)] and $\mathrm{CH}_{2-3}$ broad triplet around $2900 \mathrm{~cm}^{-1}$ [Liu (1997), Kulisch (2006), Marcinauskas, JOAM (2010)]. The intensity ratio $\mathrm{C}=\mathrm{C}\left(1610 \mathrm{~cm}^{-1}\right) / \mathrm{CO}\left(1756 \mathrm{~cm}^{-1}\right)$ increases from 0.96 to 1.03 whereas the intensity ratio $\mathrm{CH}_{\mathrm{x}}\left(1437 \mathrm{~cm}^{-1}\right) / \mathrm{C}=\mathrm{C}\left(1610 \mathrm{~cm}^{-1}\right)$ decreases from 1.00 to 0.83 when the Plexiglas box is filled with argon. The evolutions of these ratios show an improvement of the chemical stability of the unsaturated carbon bonds due to $\mathrm{O}_{2}$ depletion in the post-discharge environment and a better decomposition of $\mathrm{C}_{2} \mathrm{H}_{2}$, promoted by the temperature increase and the extension of the plasma.

Two specific micro-patterns were deposited under maximal Ar inerting flow rate $\left(1 \% \mathrm{Rh}-0.1 \% \mathrm{O}_{2}\right)$ : a straight line $(1.3 \mu \mathrm{m} \times 4 \mathrm{~mm})$ and a square $\left(50 \times 50 \mu \mathrm{m}^{2}\right)$ with a displacement velocity of $100 \mu \mathrm{m} . \mathrm{s}^{-1}$. The size of the capillary was $5 \mu \mathrm{m}$ in diameter. AFM images are presented in figure 9: 

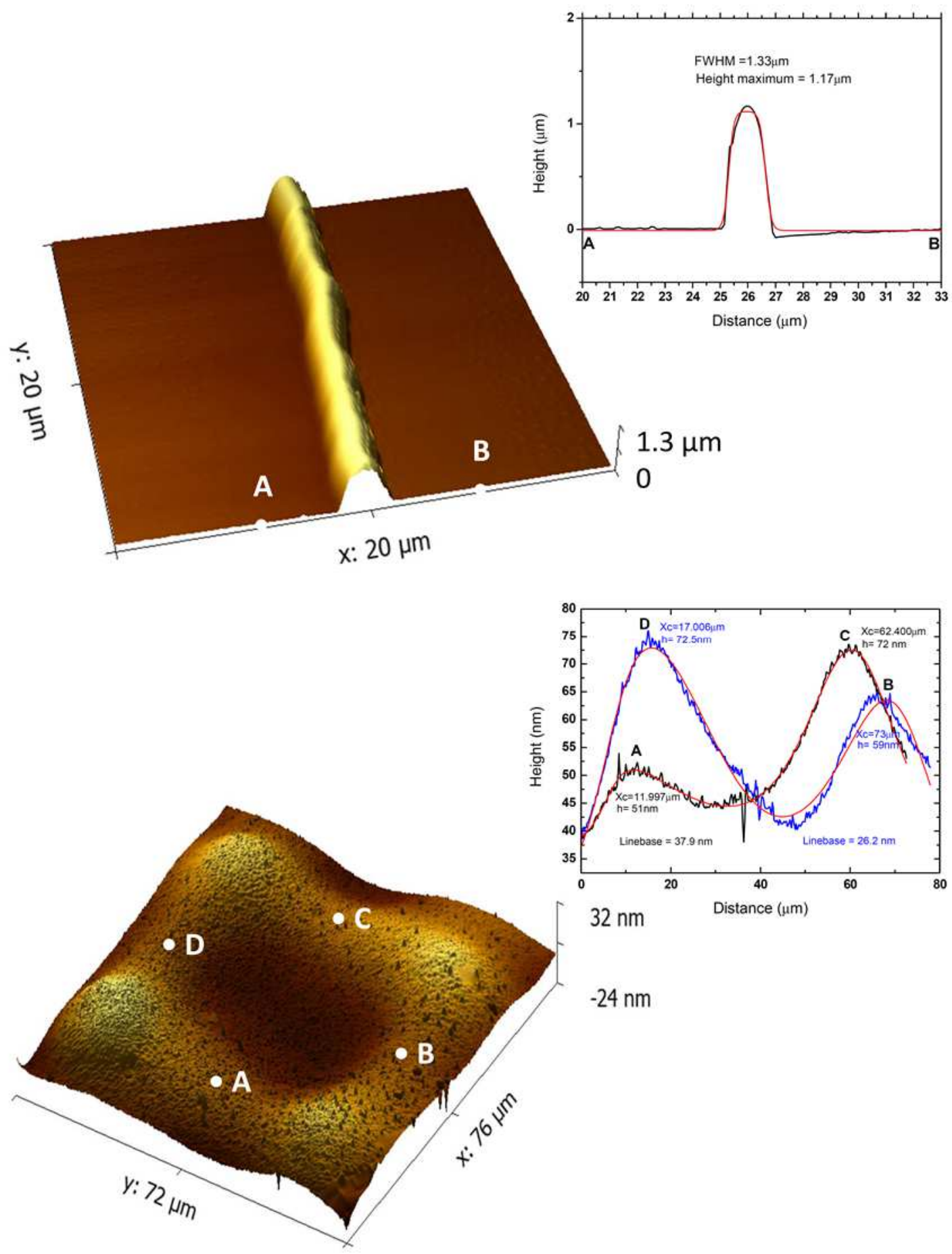

Figure 9. AFM images of a hydrocarbon line and a hydrocarbon square deposited under argon inerting conditions ( $\left.1 \% \mathrm{Rh}-0.1 \% \mathrm{O}_{2}\right)$.

In the case of the straight line (figure 9a), the pattern shows a good linearity with smallscale oscillations, which can be attributed to low hydrodynamic fluctuations in the plasma 
flow rate. The AFM image was recorded at the beginning of the line. In the investigated area, the full width at half maximum (FWHM) is found to be closed to $1.33 \mu \mathrm{m}$ and this value is constant along the entire range of the line $(\sim 2 \mathrm{~mm})$. The deposition rate is estimated at $\sim 1200$ $\mathrm{nm} . \mathrm{s}^{-1}$. The FWHM is slightly lower than the inner capillary diameter $(5 \mu \mathrm{m})$. The lower width of the line with regard to the capillary diameter is attributed to the etching mechanism induced by atomic oxygen and to a lesser extent by $\mathrm{H}$, as evidenced by OES measurements. This observation is confirmed by the deposition of the square pattern (figure 9b). The calculated average growth rate is $900 \mathrm{~nm} \cdot \mathrm{s}^{-1}$. This estimation is closed to growth rate estimated for the straight line. The AFM image and the extracted thickness profiles presented in insert of figure $9 \mathrm{~b}$, reveal that the four sides are not equivalent. A progressive decrease in the height of the structure appears over time. Consequently, the progressive lowering of the height is also attributed to the etching of the carbon. These results show that a small amount of oxygen is sufficient to etch a localized coating. The etching rate calculated from the extracted AFM thickness profiles is $22.5 \mathrm{~nm} . \mathrm{s}^{-1}$. This value is much lower than the deposition rate but the displacement velocity is very fast and the coating left exposed in post-discharge is submitted to an efficient etching. In the perspective of further miniaturization, small oxygen contamination should be completely eliminated.

\section{Conclusion}

In summary, we have successfully demonstrated the ability to deposit submicronic amorphous hydrogenated carbon spots onto a silicon surface. The best spot resolution is obtained at $3 \mathrm{~mm}$ from the discharge, in the post-discharge region. This distance corresponds to the place where the reactive species $\mathrm{O}$ and $\mathrm{OH}$ involved in the $\mathrm{C}_{2} \mathrm{H}_{2}$ dissociation are formed by dissociation in the plasma of $\mathrm{O}_{2}$ and $\mathrm{H}_{2} \mathrm{O}$ coming from the surrounding medium. 
Next, deposition under atmospheric conditions shows that hydrocarbon patterns are subjected to a subsequent dewetting phenomenon due to post-hydrolysis of $\mathrm{C}-\mathrm{O}$ surface moieties probably activated by reaction of free radicals embedded in the a:C:H matrix with the molecular oxygen. In the case of deposition in static condition, the post-dewetting phenomenon enhances the spot localization but for lines or more advanced geometries, the post-dewetting destroys the deposited shapes after treatment. In the next step, we have shown that argon inerting of surrounding medium avoids the post-dewetting and greatly improves the patterning of micrometric lines. Also, we have shown that the gases free from impurities have to be controlled with the greatest possible care. In these inerting conditions, micrometric a$\mathrm{C}: \mathrm{H}$ lines and squares were deposited onto silicon with a micrometric resolution never reached before by APPJs. Finally, this additive manufacturing process based on a decoupled method using an APPJ and a precursor micro or nano-jet offers an unusually high resolution at low cost.

\section{Acknowledgments}

The authors wish to thank P. Grysan and P. Choquet from the Luxembourg Institute of Science and Technology (LIST) for their assistance on AFM measurements and their interest in this work. 


\section{Supplemental material}

The study of the jet flow requires the calculation of several hydrodynamic parameters that depend on the capillary geometry and the acetylene upstream pressure imposed into the capillary. First, the acetylene flow rate $Q$ (expressed in $\mathrm{m}^{3} \cdot \mathrm{s}^{-1}$ ) at the capillary outlet can be calculated according to the following relation:

$$
Q=\frac{S_{B}}{\sqrt{1-\frac{D_{B}}{D_{A}}}} \cdot \sqrt{\frac{2 \cdot\left(P_{A-} P_{B}\right)}{\rho}}
$$

Where $S_{B}$ is the surface of the extremity aperture in $\mathrm{m}, D_{B}$ is the diameter of this aperture, $D_{A}$ is the inner diameter of the non-forged capillary $\left(0.64 .10^{-3} \mathrm{~m}\right), P_{A}$ is the upstream pressure imposed in the capillary in $\mathrm{Pa}, P_{B}$ is the standard atmospheric pressure (101325 Pa) and $\rho$ is the volume weight of acetylene taken at $1.11 \mathrm{~kg} \cdot \mathrm{m}^{-3}$.

Then, the jet velocity can be calculated as follows,

$$
v=\frac{4 Q M P_{A}}{\rho \pi D_{B}^{2} R T}
$$

Where $M$ is the acetylene molar weight (in $\mathrm{kg} \cdot \mathrm{mol}^{-1}$ ), $R$ is the constant of ideal gases and $T$, the temperature of the gas.

Knowing the jet velocity, the Reynolds number Re can be expressed as [Arnoult (2008)]:

$$
R e=\frac{\rho v D_{A}}{\mu(T)}
$$

The dynamic viscosity of acetylene expressed in $\mu \mathrm{Pa} . \mathrm{s}$ is estimated using the relation $\mu(T)=1.267+0.0305 T$ obtained from data reported in the reference [CRC (2006)]. 
The sound velocity at the temperature $T$ is calculated from the ideal gas law:

$$
v_{S}=\sqrt{\gamma \frac{R T}{M}}
$$

It is useful to note that $\gamma=5 / 3$ for a monoatomic gas and $\gamma=7 / 5$ for a diatomic gas. The $\gamma$ coefficient $5 / 3$ is usually used for precursor diluted in argon. However, in our case, $\gamma$ must be calculated as the ratio between the isobaric and the isochoric heat capacity at the temperature of the jet in the post-discharge. The calculation of the $\gamma$ coefficient established on the basis of $C p(T)=4.16608 .10^{-2} \mathrm{~kJ} \cdot \mathrm{mol}^{-1}$ and $C v(T)=3.30682 .10^{-2} \mathrm{~kJ} \cdot \mathrm{mol}^{-1}$ data for acetylene gives $\gamma=1.2598$. This value is close to the $\gamma$ coefficient for a diatomic gas. Eventually, the sound velocity is estimated at $580 \mathrm{~m} \cdot \mathrm{s}^{-1}$ for a gas temperature of $450 \mathrm{~K}$ (assuming that thermal equilibrium is reached rapidly). 


\section{References}

[Maruo (1997)] S. Maruo, O. Nakamura and S. Kawata, Optics Letters, 22, 2, 132-134 (1997).

[Cumpston (1999)] B. H. Cumpston, S. P. Ananthavel, S. Barlow, D. L. Dyer, J. E. Ehrlich, L. L. Erskine, A. A. Heikal, S. M. Kuebler, I.-Y. Sandy Lee, D. McCord-Maughon, J. Qin, H. Röckel, M. Rumi, X.-L. Wu, S. R. Marder and J. W. Perry, Nature, 398 (1999).

[Kawata (2001)] S. Kawata, H.B. Sun, T. Tanaka, K. Takada, Nature, 412 (2001).

[Serbin (2003)] J. Serbin, A. Egbert, A. Ostendorf, and B. N. Chichkov, Optics Letters, 28, 5, 301-303 (2003).

[Deubel (2004)] M. Deubel, G. Von Freymann, M Wegener, S. Pereira, K. Busch and C. M. Soukoulis, Nature Materials, 3 (2004).

[Mariottti, (JPD 2011)] D. Mariotti and R. Mohan Sankaran, J. Phys. D: Appl. Phys., 44, $174023(2011)$.

[Belmonte (JPD 2011)] T. Belmonte, G. Arnoult, G. Henrion and T. Gries, J. Phys. D: Appl. Phys. 44, 363001 (2011).

[Belmonte (JTST 2011)] T. Belmonte, G. Henrion, and T. Gries, Journal of Thermal Spray Technology, 20, 4, 744-759 (2011).

[Motrescu (2012)] I. Motrescu, A. Ogino and M Nagatsu, J. of Photopolymer Science and Technology, 25, 4, 529-534 (2012).

[Hollander (2003)] A. Holländer and L. Abhinandan, Surface and Coatings Technology 174175, 1175-1177 (2003).

[Abhinandan (2004)] L. Abhinandan and A. Holländer, Thin Solid Films 457, 241-245 (2004). 
[Kakei (2010)] R. Kakei, A. Ogino, F. Iwata and M. Nagatsu, Thin Solid Films 518, 34573460 (2010).

[Shimizu (2003)] Y. Shimizu, T. Sasaki, T. Ito, K. Terashima and N. Koshizaki, J. Phys. D: Appl. Phys., 36, 2940-2944 (2003).

[Miyake (1987)] S. Miyake, S. Takahashi, I. Watanabe, H. Yoshihara, ASLE Trans., 30, 1, 121 (1987).

[Zaidi (1994)] H. Zaidi, T. Le Huu, D. Paulmier, Diamond Relat. Mater., 3, 1028-1033 (1994).

[Fontaine (2001)] J. Fontaine, C. Donnet, A. Grill, T. LeMogne, Surface and Coatings Technology $146-147,286-291$ (2001).

[Comprehensive Hard Materials (2014)] V. K. Sarin, Comprehensive Hard Materials, p. 125 (2014).

[Robertson 1998] J. Robertson, in: S.R.P. Silva et al. (Eds.), Amorphous Carbon : State of the Art, World Scientific, Singapore (1998).

[Robertson 2002] J. Robertson, Materials Science and Engineering R, 37, 129-281 (2002).

[Chen (2013)] X. Chen, T. Kato, M. Kawaguchi, M. Nosaka and J. Choi, J. Phys. D: Appl. Phys., 46, 255304 (2013).

[Manage in thesis (1998)] D. P. Manage, Structural and Optical characterization of hydrogenated amorphous thin films, Thesis, University of Toronto (1998).

[Manage (2000)] D. P. Manage, J. M. Perz, F. Gaspari, E. Sagnes, S. Zukotynski, J. of NonCrystalline Solids, 270, 247-254 (2000). 
[Eden (2006)] J. G. Eden and S.-J. Park, Phys. Plamas, 13, 057101 (2006).

[Mariotti (JPD 2010)] D. Mariotti and R. Mohan Sankaran, J. Phys. D: Appl. Phys., 43, 323001 (2011).

[Seo (2010)] Y. S. Seo, A-A. H. Mohamed, K. C. Woo, H. Wook Lee, J. K. Lee, IEEE Transactions on plasma science, 93, 3813 (2010).

[Fauchais (1997)] P. Fauchais, A. Vardelle, A. Denoirjean, Surf. Coat. Technol., 97, 66-78 (1997).

[Asmussen (2002)] J. Asmussen, D. Reinhard, Diamond Thin Films Handbook, Marcel Dekker Incorporated, New York (2002).

[Benedikt (2002)] J. Benedikt, K. G. Y. Letouneur, M. Wisse, D. C. Schram, M. C. M. van de Sanden, Diamond Relat. Mater., 11, 989-993 (2002).

[Pothiraja (2012)] R. Pothiraja, N. Bibinov and P. Awakowicz, Chemical Kinetics, Dr Vivek Patel (Ed.), ISBN: 978-953-51-0132-1, InTech, 227-252 (2012).

[Bussiahn (2010)] R. Bussiahn, E. Kindel, H. Lange and K. D. Weltmann, J. Phys. D: Appl. Phys., 43, 165201 (2010).

[Yu (2006)] Q. S. Yu, C. Huang, F. H. Hsieh, H. Huff and Y. Duan, J. of Biomedical Materials Research Part B: Applied Biomaterials, 211 - 219 (2006).

[Xiong (2010)] Q. Xiong, A. Y. Nikiforov, X. P. Lu and C. Leys, J. Phys. D: Appl. Phys. 43, $415201(2010)$.

[Leblond (1981)] J. B. Leblond, F. Collier, F. Hoffbeck, and P. Cottin, J. Chem. Phys. 74, 11 (1981). 
[Jeong (1998)] J. Y. Jeong, S. E. Babayan, V. J. Tu, J. Park, I. Henins, R. F. Hicks and G. S. Selwyn, Plasma Sources Sci. Technol., 7, 282-285 (1998).

[Jeong (1999)] J. Y. Jeong, S. E. Babayan, A. Schütze, V. J. Tu, J. Park, I. Henins, G. S. Selwyn and R. F. Hicks, J. Vac. Sci. Technol. A 17, 5, 2581-2585 (1999).

[Lu (2008)] X. P. Lu, T. Ye, Y. G. Cao, Z. Y. Sun, Q. Xiong, Z. Y. Tang, Z. L. Xiong, J. Hu, Z. H. Jiang and Y. Pan, J. of Applied Physics, 104, 053309 (2008)

[Onno (2000)] R. Ono and T. Oda, IEEE Transactions on industry applications, 36, 1, 82-86 (2000).

[Luna-Lopez (2009)] J. A. Luna-López, J. Carrillo-López, M. Aceves-Mijares, A. MoralesSánchez and C. Falcony, Superficies y Vacío, 22, 1, 11-14 (2009).

[Konshina (1997)] E. A. Konshina, V. A. Tolmachev, A. L. Vangonen and L. A. Fatkulina, J. Opt. Technol., 64, 476 (1997).

[Marcinauskas (JOAM 2010)] L. Marcinauskas, A. Grigonis, P. Valatkevičius, J. of Optoelectronics and Advanced Materials, 12, 4, 829 - 833 (2010).

[Mawhinney (2001)] D. B. Mawhinney and J. T. Yates Jr., Carbon, 39, 1167-1173 (2001).

[Fanning (1993)] P. E. Fanning and M. A. Vannice, Carbon, 31, 721 (1993).

[Theil (1990)] J. A. Theil, D. V. Tsu, M. W. Watkins, S. S. Kim, and G. Lucovsky, Journal of Vacuum Science \& Technology A, 8, 1374 (1990).

[Liu (1997)] S. Liu, S. Gangopadhyay, G. Sreenivas, S. S. Ang and H. A. Naseem, Physical Review B, 55, 19, 13020-13024 (1997).

[Kulisch (2006)] W. Kulisch, C. Popov, S. Boycheva, M. Jelinek, P. N. Gibson, V. Vorlicek, Surface \& Coatings Technology, 200, 4731 - 4736 (2006). 
[Robertson (1991)] J. Robertson, Prog. Solid State Chem., 21, 199 (1991).

[Salonen (2004)] J. Salonen, M. Björkqvist, E. Laine and L. Niinistö, Appl. Surface Science, 225, 389-394 (2004).

[Smith (1995)] D.M. Smith and A.R. Chughtai, Colloids and Surfaces A: Physicochemical and Engineering Aspects, 105, 47-77 (1995).

[Nguyen (JPC 1995)] M. T. Nguyen, D. Sengupta, G. Raspoet and L. G. Vanquickenborne, J. Phys. Chem., 99, 11883-11888 (1995).

[Valor (2002)] A. Valor, E. Reguera, E. Torres-García, S. Mandoza and F. Sanchez-Sinencio, Thermochimica Acta, 389, 133-139 (2002).

[Criegee (1975)] R. Criegee, Angew. Chem. Internal. Edit., 14, 1, 11, 745-752 (1975).

[Neeb (1996)] P. Neeb, O. Horie and G. K. Moortgat, Tetrahedron Letters, 37, 52, 9297-9300 (1996).

[Neeb (1998)] P. Neeb, O. Horie and G. K. Moortgat, J. Phys. Chem. A, 102, 6778-6785 (1998).

[Manoilova (1998)] O. V. Manoilova, J. C. Lavalley, N. M. Tsyganenko, and A. A. Tsyganenko, Langmuir, 14, 5813-5820 (1998).

[Bilek (2011)] M. M. M. Bilek, D.1 V. Bax, A. Kondyurina, Y. Yin, N. J. Nosworthy, K. Fisher, A. Waterhouse, A. S. Weiss, C. G. dos Remedios and D. R. McKenzie, Proceedings of the National Academy of Sciences, 108, 35, 14405-14410 (2011).

[Kosobrodova (2012)] E. A. Kosobrodova, A. V. Kondyurin, K. Fisher , W. Moeller, D. R. McKenzie, M. M. M. Bilek, Nuclear Instruments and Methods in Physics Research B, 280, 26-35 (2012). 
[Kosobrodova (2014)] E. A. Kosobrodova, A. V. Kondyurin , W. Chrzanowski, D.G. McCulloch, D. R. McKenzie, M. M. M. Bilek, Nuclear Instruments and Methods in Physics Research B, 329, 52-63 (2014).

[Pearse 1976] R. W. B. Pearse and A. G. Gaydon, The Identification of molecular spectra, $4^{\text {th }}$ Edition (1976).

[Kutasi (2011)] K. Kutasi, V. Guerra and P. A. Sà, Plasma Sources Sci. Technol. 20, 035006 (2011).

[Louh (2006)] S. P. Louh, C. H. Wong and M. H. Hon, Thin Solid Films, 498, 235-239 (2006).

[Marcinauskas Vacuum (2007)] L. Marcinauskas, A. Grigonis, V. Kulikauskas and V. Valincius, Vacuum, 81, 1220-1223 (2007).

[Arnoult (2008)] G. Arnoult, R. P. Cardoso, T. Belmonte and G. Henrion, Applied Physics Letters, 93, 191507 (2008).

[CRC (2006)] D. R. Lide, CRC handbook of chemistry and physics: a ready-reference book of chemical and physical data : 2006-2007; CRC Press: Boca Raton (2006). 